\title{
ANALYZING ASTRONOMICAL OBSERVATIONS WITH THE NASA AMES PAH DATABASE
}

\author{
J. Cami ${ }^{1,2}$
}

\begin{abstract}
We use the NASA Ames Polycyclic Aromatic Hydrocarbon (PAH) infrared spectroscopic database to model infared emission of PAHs following absorption of a UV photon. We calculate emission spectra resulting from the full cooling cascade for each species in the database. Using a least squares approach, we can find out what PAH mixtures best reproduce a few typical astronomical observations representing the different classes of UIR spectra. We find that we can reproduce the observed UIR spectra in the wavelength range 6-14 $\mu \mathrm{m}$, offering support for the hypothesis that the UIR bands are indeed due to vibrational modes of PAHs and related molecular species. Spectral decompositions of our best fit models confirm and reinforce several earlier results: (i) the $6.2 \mu \mathrm{m}$ band requires a significant contribution of nitrogen-substituted PAHs (PANHs); (ii) the reported components and their variations in the $7.7 \mu \mathrm{m}$ band are indicative of changes in the size distribution of the contributing molecules; (iii) there is a significant contribution of anions to the $7.7 \mu \mathrm{m}$ band; (iv) the $11.2 \mu \mathrm{m}$ band is due to large, neutral and pure PAHs; $(v)$ the $11.0 \mu \mathrm{m}$ band is due to large PAH cations.
\end{abstract}

\section{Introduction}

The PAH hypothesis that was introduced 25 years ago states that the so-called Unidentified InfraRed (UIR) bands result from PAH molecules that are transiently heated by absorption of a UV photon, and subsequently cool by emission of infrared photons through vibrational relaxation (IR fluorescence). Although this hypothesis has now been generally accepted by the astronomical community, a good and detailed spectral match between a PAH mixture and astronomical observations has not yet been produced. At the same time, it is not clear exactly

\footnotetext{
1 Department of Physics and Astronomy, University of Western Ontario, London, Ontario N6A 3K7, Canada

2 SETI Institute - 189 Bernardo Ave., Suite 100 - Mountain View, CA 94043, USA
} 
what the properties are of the astronomical PAH population. Such information is vital for astronomers if they are to use the widely observed UIR bands as diagnostic tools for probing the wide variety of astrophysical environments in which the UIR bands are observed.

Here, we present the first results of a program that uses the NASA Ames PAH infrared spectroscopic database to analyze astronomical observations. We show that a mixture of PAHs can indeed reproduce the observed UIR emission bands and their spectral variations. Furthermore, we gain some insight into the nature of astronomical PAHs from spectral decompositions of the best fit models.

\section{Modeling the PAH emission}

\subsection{PAH species}

The NASA Ames PAH IR spectroscopic database (Bauschlicher et al. 2010; see also Boersma et al. elsewhere in this volume) is currently the largest collection of infrared properties of PAHs and some related molecules (e.g. the nitrogensubstituted PANHs, see Hudgins et al. 2005). The database contains theoretically calculated properties for a much larger set of molecules than is experimentally available; here, we used the theoretical database exclusively.

Amongst others, the theoretical part of the database contains the calculated frequencies and intensities for all the fundamental mode vibrations for a collection of 556 individual species. The database is highly biased toward smaller $(\leq 30 \mathrm{C}$ atoms) and pure PAHs that are neutral or singly ionized. We show in Section 4 that reliable conclusions can still be inferred from such a biased database.

\subsection{PAH physics}

It is well established (see e.g. d'Hendecourt et al. 1989) that the PAH excitation and IR fluorescent cooling can be adequately described by using the thermal approximation which states that the vibrational energy contained in a large, isolated molecule can be represented by a single parameter - the vibrational excitation temperature. Absorption of a single UV photon will then briefly heat a molecule to very high temperatures, after which it cools by infrared emission that scales with the Planck function at the vibrational temperature (see also Allamandola et al. 1989). The total PAH emission following absorption of a single UV photon is then the time-integrated infrared emission as the molecule cools down.

The maximum temperature that can be attained by a $\mathrm{PAH}$ molecule after UV absorption depends on the energy of the absorbed photon, and on the molecule capability of distributing the energy over the vibrational states - the "vibrational" heat capacity. Since larger molecules have more vibrational modes, the average energy per vibrational mode is lower for a given photon energy and thus the larger molecules have typically lower temperatures after absorption of the same photon. As a consequence, they will emit much of their energy at longer wavelengths than the smaller PAHs. 
To take such effects properly into account, we used the data in the PAH database to first calculate the heat capacities for each individual species. We also calculated the maximum temperature for each species upon absorption of a $10 \mathrm{eV}$ photon, and calculated the cooling rate for each species for temperatures between $0 \mathrm{~K}$ and the maximum attained temperature (see e.g. Tielens 2005). This then allows to calculate the full fluorescent infrared emission for each molecule in the database by integrating the emission over the cooling curve. A check is performed to ensure energy conservation in the process.

\subsection{PAH parameters}

The PAH physics described above thus sets the relative intensities for all fundamental modes for each molecular species individually, and the only free parameter is the energy of the absorbed UV photon. However, in order to compare these model spectra to astronomical observations, we need to adopt a band profile shape. Most of the astronomical UIR bands are characterized by asymmetric band profiles that are believed to originate from anharmonicities in the potential energy. This in turn results in hot band transitions that are slightly shifted in frequency compared to the fundamental, and causes the overall profile to be asymmetric.

Since these effects reflect the details of the potential energy surface, they are generally different for each vibrational mode and for each molecule, and the resulting band asymmetry is furthermore a function of the temperature. Unfortunately, the PAH database does not contain information on anharmonicity and integrating anharmonic effects is a very demanding task that is restricted so far to a few small species (see Pirali et al. 2009; Basire et al. elsewhere in this volume). We thus decided to convolve each of the individual PAH bands with a symmetric Lorentzian profile. We acknowledge that this will necessarily introduce some uncertainty in the wings of the PAH bands; and we will therefore not discuss spectral features at that level.

Finally, we only consider absorption by photons of one single energy - the average photon energy for the environment studied. The above procedures thus result in a set of 556 individual spectra, representing the response of each species in the database to absorption by a photon with the same energy.

\section{Fitting the astronomical spectra}

\subsection{Observations}

Peeters et al. (2002) and van Diedenhoven et al. (2004) have shown that most astronomical observations of the PAH bands can be divided in only a few classes (Class A, B and C; see also Peeters et al. elsewhere in this volume). Whereas objects in class A have identical spectra, classes B and C represent a much larger variety in terms of peak positions of individual features and relative intensities of the PAH bands. For this work, we selected a representative object for class A (the HII region IRAS $23133+6050$ ), but somewhat arbitrarily picked an object from 

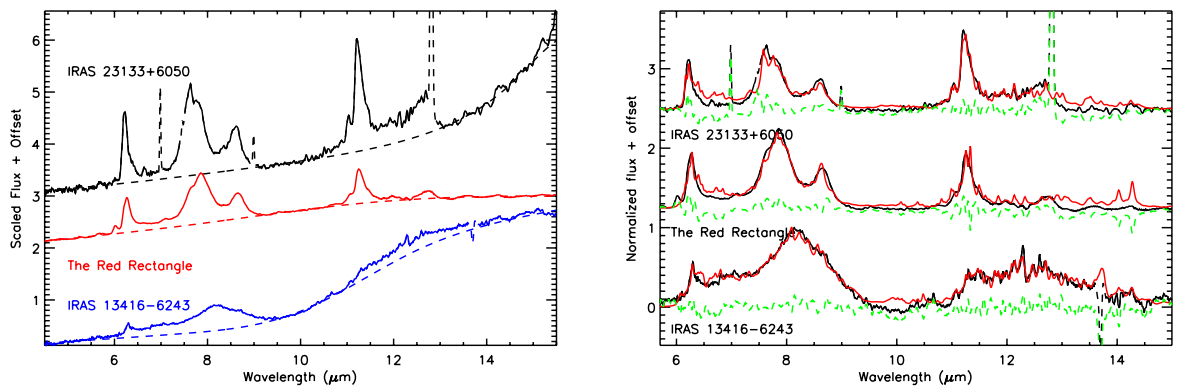

Fig. 1. (a) The three astronomical observations (solid lines) and the adopted dust continua (dashed lines). The Hit region IRAS 23133+6050 (top) is a Class A source; the Red Rectangle (middle) a Class B source and the proto-planetary nebula IRAS 13416-6243 (bottom) a Class C object. (b) The astronomical PAH spectra (black) and the best fit PAH model spectra (ligther curves). Residuals are shown as dashed lines.

class B (the Red Rectangle) and another one from class C (the proto-planetary nebula IRAS 13416-6243). If we can reproduce the observable UIR properties for these three objects with PAHs, it stands to reason that we can equally well reproduce most other observations.

All observations used here are ISO/SWS observations; we refer to Peeters et al. (2002) for details about the data reduction process. We do note here however that we chose to define a continuum that only represents the thermal emission of dust, and thus does not contain any spectral features to represent possible plateaus underneath the PAH features (see Fig. 1a). For what follows, we will always use the continuum-subtracted spectra.

\subsection{Procedures}

We calculated individual PAH spectra for each of the species in the database as described above, using an average photon energy of $10 \mathrm{eV}$. Then, we used a nonnegative least squares algorithm to determine "abundances" (weights) for each of those PAHs such that the weighted sum of the PAH spectra provides the best possible fit to each of these astronomical observations.

One issue that needs to be addressed here is the intrinsic widths for each of the $\mathrm{PAH}$ bands in our models. Often, it is assumed that the intrinsic PAH bands are fairly broad $\left(\sim 30 \mathrm{~cm}^{-1}\right)$. However, the astronomical observations of the strong $\mathrm{C}-\mathrm{H}$ out of plane bending mode at $11.2 \mu \mathrm{m}$ show a band width (FWHM) of only about $4 \mathrm{~cm}^{-1}$. Obviously, the constituent PAH spectra that make up this band cannot be broader than this observed width. The same holds at least for the band at $11.0 \mu \mathrm{m}$. In the $6-10 \mu \mathrm{m}$ region on the other hand, the observed band profiles are broader (in wavenumber space), and it thus seems unrealistic to adopt a small band width for the PAH profiles in this wavelength range. With some trial and 

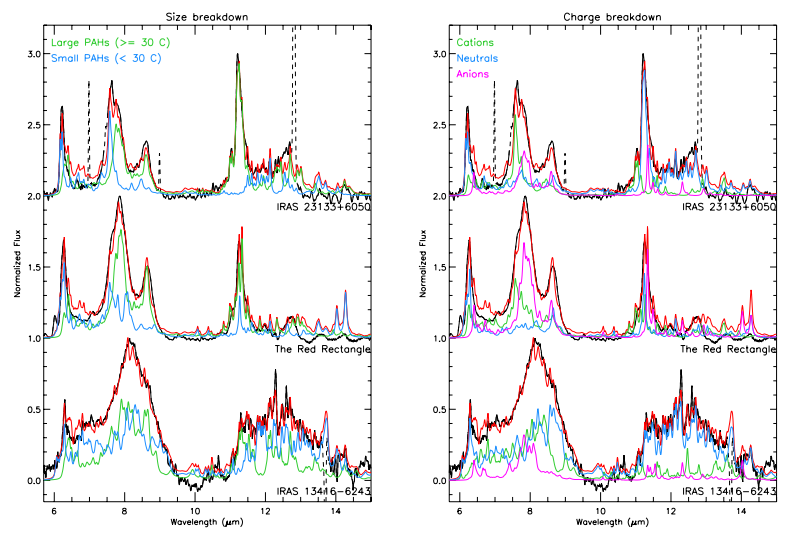

Fig. 2. The spectral decomposition of our best fitting model as a function of molecular size (left) and charge state (right). Astronomical observations are shown in black; overall fits in red, and decompositions in green, blue or magenta (see legend).

error, we found that in this region, a width (FWHM) of $13 \mathrm{~cm}^{-1}$ provides better global results. Thus, we convolved the individual PAH spectra with a Lorentzian profile that has a FWHM of $13 \mathrm{~cm}^{-1}$ in the $6-10 \mu \mathrm{m}$ region, and a width of $4 \mathrm{~cm}^{-1}$ in the 10-14 $\mu \mathrm{m}$ region. The resulting best fits are shown in Figure 1b.

\section{Results \& discussion: Fits \& decomposition}

Figure 1b clearly shows that a mixture of PAHs can indeed reproduce the observed infrared emission for all three classes of astronomical PAH spectra. Typically, about 50 different molecular species are included in the models presented here.

Much more valuable than the fits themselves is the insight we can gain into the nature of the astrophysical PAH population by breaking down our best fit models into spectral decompositions.

Figure 2 shows just two such examples. In the left panel, the green curve represents the contribution to our total fit of the "large" PAHs, i.e. the species in our database with 30 or more carbon atoms; the blue curve represents the small PAHs. This figure shows several interesting results. First, for both the Class A and B sources, the strong $11.2 \mu \mathrm{m}$ PAH band is (almost) exclusively due to large PAH species. This is consistent with the band width of $4 \mathrm{~cm}^{-1}$ that implies a mean excitation temperature of about $280 \mathrm{~K}$ (Pech et al. 2002) and thus excludes a significant contribution of small PAHs that are too hot. Note that the NASA Ames PAH database is currently heavily biased toward smaller species, and given that bias, it is somewhat surprising to find that a spectral feature is exclusively due to large PAHs. Clearly, not a single one of the hundreds of small PAH species in the database is able to reproduce the $11.2 \mu \mathrm{m}$ band without causing additional spurious spectral features in this wavelength range. Also the PAH bands at 8.6 
and $11.0 \mu \mathrm{m}$ are due to large PAHs, and the same holds for the red component of the $7.7 \mu \mathrm{m}$ complex. Variations in the shape of the $7.7 \mu \mathrm{m}$ complex are then indicative of changes in the size distribution of the PAHs. Note also that the Class $\mathrm{C}$ source contains what appears to be a random distribution of both large and small PAHs.

In the right panel, the best fit is decomposed according to the charge state of the contributing species: neutrals are shown in blue, cations in green, and anions in magenta. Neutral PAHs produce the bulk of the emission in the $11.2 \mu \mathrm{m}$ band, but cations are responsible for the $11.0 \mu \mathrm{m}$ band. Combined with the left panel, we can thus conclude that the $11.2 \mu \mathrm{m}$ band is due to large neutral PAHs, while the $11.0 \mu \mathrm{m}$ band is due to large PAH cations. It is also interesting to see that our best model includes a significant fraction of anions in the mixture. This is especially clear in the $7.7 \mu \mathrm{m}$ complex. Finally, note that for the class $\mathrm{C}$ source, most of the PAH emission arises from neutral molecules.

Many more spectral decompositions are possible, and yield interesting results. One in particular is a decomposition according to the chemical composition which shows that the $6.2 \mu \mathrm{m}$ band requires a significant contribution of nitrogen-substituted PAHs (called PANHs) - as was shown already by Hudgins et al. (2005).

The work presented here illustrates the analytic and diagnostic power that is contained in large spectral databases such as the NASA Ames PAH IR spectroscopy database. The fact that some of our results from the spectral decomposition analysis go against the bias in the database (e.g. exclusively large PAHs in the results while the database contains primarily small PAHs) indicates that our results are not driven by the database contents, but rather by the spectral properties of the astronomical PAHs. At the same time, we are somewhat limited by the relatively small number of molecules in the database, and by the uncertainties in the intrinsic band profiles. Nevertheless, the NASA Ames PAH database clearly contains already a significant number of astrophysically relevant PAH species, and future research will undoubtedly increase the diagnostic value of exercises such as the one presented here.

\section{References}

Allamandola, L.J., Tielens, A.G.G.M., \& Barker, J.R., 1989, ApJS, 71, 733

Bauschlicher, C.W., Boersma, C., Ricca, A., et al., 2010, ApJS, 189, 341

d'Hendecourt, L., Léger, A., Boissel, P., \& Désert, F., 1989, IAUS, 135, 207

Hudgins, D.M., Bauschlicher, C.W. Jr., \& Allamandola, L.J., 2005, ApJ, 632, 316

Pech, C., Joblin, C., \& Boissel, P., 2002, A\&A, 388, 639

Peeters, E., Hony, S., van Kerckhoven, C., et al., 2002, A\&A, 390, 1089

Pirali, O., Vervloet, M., Mulas, G., Malloci, G., \& Joblin, C., 2009, Phys. Chem. Chem. Phys., 11, 3443

Tielens, A.G.G.M., 2005, The Physics and Chemistry of the Interstellar Medium (Cambridge University Press)

van Diedenhoven, B., Peeters, E., van Kerckhoven, C., et al., 2004, ApJ, 611, 928 